\title{
The Drosophila TNF ortholog Eiger: Emerging physiological roles and evolution of the TNF system
}

Tatsushi Igaki $^{\mathrm{ab}^{*}}$, Masayuki Miura ${ }^{\mathrm{cd}^{*}}$

${ }^{a}$ Laboratory of Genetics, Graduate School of Biostudies, Kyoto University,

Yoshida-Konoecho, Sakyo-ku, Kyoto 606-8501, Japan

${ }^{\mathrm{b}}$ Precursory Research for Embryonic Science and Technology (PRESTO), Basic

Research Program, Japan Science and Technology Agency, Chiyoda-ku, Tokyo 102-0075, Japan

${ }^{c}$ Department of Genetics, Graduate School of Pharmaceutical Sciences, The University of Tokyo, Bunkyo-ku, Tokyo 113-0033, Japan

${ }^{\mathrm{d}}$ Core Research for Evolutional Science and Technology (CREST), Basic Research

Program, Japan Science and Technology Agency, Chiyoda-ku, Tokyo 102-0075, Japan

*Corresponding authors

Tel: +81-75-753-7684

E-mail address: igaki@lif.kyoto-u.ac.jp (T. Igaki)

Tel: $+81-3-5841-4860$

E-mail address: miura@molo.f.u-tokyo.ac.jp (M. Miura)

Keywords: TNF, TNFR, JNK, Drosophila, cancer, cell death, inflammation 


\section{ABSTRACT}

The TNF and TNFR superfamilies of proteins are conserved throughout evolution.

The first invertebrate orthologs of TNF and TNFR, Eiger and Wengen, were identified

in Drosophila, which enabled us to take advantage of its powerful genetics. Indeed, genetic studies on Eiger in the last decade have discovered their signaling mechanisms through activation of the JNK pathway and unveiled the role of Eiger-JNK signaling in a variety of cellular and tissue processes such as cell death, cell proliferation, tissue growth regulation, host defense, pain sensitization, and canalization. In this review, we will describe the in vivo signaling of Eiger and its physiological roles in fly development and homeostasis, and will discuss the evolution of the TNF/TNFR systems. 


\section{Introduction}

Many of the intra- and inter-cellular signaling pathways that regulate a variety

of cellular processes such as cell proliferation, cell differentiation, cell survival, and cell

death are highly conserved throughout evolution. Thus, accumulating studies using model organisms have made a great contribution to our knowledge of the basic principles as well as evolutionary variations of cellular signaling. Drosophila provides a powerful genetic model for studying the in vivo roles of the signaling pathways and their physiological regulations. Drosophila has the member of the tumor necrosis factor (TNF) Eiger and its receptor Wengen [1-3]. Genetic studies in Drosophila have identified evolutionarily conserved molecular functions of TNF family proteins and are now providing new dimensions toward understanding their physiological roles and evolution.

\section{Drosophila TNF ortholog Eiger, a ligand for JNK signaling}

The Drosophila ortholog of TNF was identified through an ectopic expression screen in Drosophila compound eye for genes that cause cell death, and was named 
eiger (ㅁda-like cell death trigger) [1]. eiger was also isolated independently by molecular approaches $[2,3]$. The genomic database of Drosophila indicates that eiger is the sole member of the TNF superfamily in flies. eiger encodes a protein of 409 amino acids with a transmembrane (TM) domain and a C-terminal TNF homology domain, which shows highest homology with a TNF family member Ectodysplasia (EDA)-A2 (28\%) [1]. Two isoforms of Eiger have been reported; one has 409 amino acids and the other isoform, Eiger-L, contains additional 6 amino acids (GESLLS) located outside the TNF homology domain $[2,3]$. Both isoforms were shown to be expressed in flies [2]. Overexpression of Eiger in imaginal discs causes cell death by activation of the c-Jun N-terminal kinase (JNK) pathway through Misshapen (Msn, JNKKKK), dTAK1 (JNKKK), Hemipterous (Hep, JNKK), and Basket (Bsk, JNK) [1, 3]. As a receptor for Eiger, the fly TNF receptor (TNFR) homolog wengen was identified through a genetic screen for mutations that suppress Eiger-induced cell death [4]. wengen encodes a transmembrane protein with a TNFR homology domain in the extracellular region, which physically interacts with Eiger's TNF homology domain, and is required for Eiger-induced cell death [4]. Epitope-tagged expression of Wengen 
in $293 \mathrm{~T}$ cells showed that Wengen is a type I transmembrane protein, like most mammalian TNFR family members [2]. It was shown that overexpression of Eiger (Eiger-S) or Eiger-L together with Wengen in S2 cells induced cell death with the same extent [2].

Intriguingly, Wengen does not possess an intracellular motif called 'death-domain', which is commonly found in the TNFR superfamily members and is required for downstream activation of caspases. This suggests that there are other mechanism(s) for Wengen to transduce signals. Indeed, subsequent genetic studies have identified the Drosophila homolog of TAB2/3 (dTAB2), which links TNF receptor-associated factor (DTRAF1) to TAK1 [5], as well as DTRAF2 and the deubiquitinating enzyme dCYLD [6], as components of the Eiger-JNK signaling pathway. dCYLD, the fly homolog of the tumor suppressor mutated in familial cylindromatosis, facilitates Eiger signaling by deubiquitination of DTRAF2, which stabilizes the DTRAF2 protein and thereby triggers the JNK pathway. Further analyses for Eiger-induced signaling have shown that Bendless and dUev1a, which form an ubiquitin-conjugating E2 enzyme complex, act downstream of Eiger to elicit 
both DTRAF2-mediated JNK-dependent cell death and NOPO (Drosophila ortholog of TRAF-interacting protein)-mediated caspase-dependent cell death [7-9] (Fig. 1).

TNF in mammals is synthesized as a membrane-anchored protein and is cleaved to generate a soluble ligand by a membrane-bound metalloproteinase called TNF-alpha converting enzyme (TACE). Although Drosophila has a gene encoding TACE homolog, it is unclear whether Eiger is processed by TACE to become a soluble protein in vivo. It has been shown that, when Eiger is expressed in Drosophila $\mathrm{S} 2$ cells, Eiger can be cleaved and secreated into the medium [2]. Furthermore, furin-like activity could cleave Eiger when expressed in 293T cells [10]. Interestingly, a recent study in Drosophila imaginal discs has shown that apoptotic cells induce additional apoptosis in the same tissue through Eiger-JNK signaling, a phenomenon named apoptosis-induced apoptosis (AiA) [11]. It was proposed that Eiger is produced in apoptotic cells and activates the JNK pathway in neighboring cells, thereby propagating the apoptotic JNK signaling in the tissue. This suggests that, under certain conditions, Eiger could be processed to become a soluble ligand and acts as a non-cell autonomous activator of JNK signaling. Such propagation of JNK activity within the imaginal disc 
was also observed upon wounding [12]. However, it was shown that, when overexpressed in imaginal discs as clones, Eiger activates JNK signaling strictly in a cell-autonomous manner [13]. Overexpression of a modified Eiger, which lacks transmembrane domain but has additional hemagglutinin signal peptide at $\mathrm{N}$-terminus (called 'soluble Eiger'), in eye imaginal disc induced eye-ablation phenotype [10]. It is unclear whether this soluble Eiger can induce cell death through Wengen. Indeed, it was reported that physical interaction between soluble Eiger and Wengen was not observed [2]. Since Wengen has only a single cysteine-rich TNFR homology domain, affinity and/or specificity to the ligand could be low [2]. It is possible that soluble Eiger-induced JNK signaling is mediated through a receptor other than Wengen.

\section{Metabolic control of Eiger signaling}

To further dissect the downstream signaling pathway of Eiger-induced cell death, an unbiased genetic screen for dominant modifiers of Eiger-induced small-eye phenotype was conducted. A series of chromosomal deficiencies that cover more than $80 \%$ of the genome were screened and responsible genes for suppressor phenotypes 
were identified using RNAi lines that target genes located within the chromosomal deletion. The screen resulted in the identification of a series of genes encoding energy production-related enzymes as suppressors of Eiger-induced cell death [14]. It was shown that metabolic enzymes act downstream of JNK to facilitate Eiger-induced cell death. Furthermore, as Eiger signaling is required for intrinsic tumor suppressor activity (see below), energy production-related enzymes were shown to play roles in such tumor suppression mechanisms. How does energy-production enzymes contribute to cell death? Clones of cells overexpressing Eiger in the eye disc were shown to be stained with dihydroethidium, which indicaties that Eiger induces production of superoxide $\left(\mathrm{O}^{2-}\right)$. In addition, a ROS-inducible gstD (glutathione $\mathrm{S}$ transferase D) promoter-GFP reporter, gstD-GFP, was induced when Eiger was ectopically expressed in the eye disc. Thus, Eiger can induce ROS production. Further genetic analyses indicated that energy production-related enzymes act upstream of ROS production, which could promote Eiger-induced cell death [14] (Fig. 1).

In mammals, some energy production-related enzymes are shown to act as tumor suppressors. For instance, succinate dehydrogenases (Sdh; SdhB, SdhC, and 
SdhD), subunits of a TCA cycle enzyme, are classical tumor suppressors in pheochromocytoma or paraganglioma [15]. A specific isoform of pyruvate kinase, which is necessary for cellular metabolism to shift to aerobic glycolysis, works to promote tumorigenesis. Knockdown of pyruvate kinase M2 (PKM2) reduced cell proliferation rate and glucose metabolism [16]. In addition, pyruvate dehydrogenase (PDH) links the glycolytic pathway to the TCA cycle by transforming pyruvate to acetyl-CoA. The activity of PDH is suppressed in cancer cells, while reactivation of PDH induces cell death in a solid tumor cell line and xenografts [17]. In Drosophia, knockdown of PDH suppressed Eiger-induced cell death [14]. These observations suggest that mitochondrial energy production could act as a tumor suppressor probably through the generation of cytotoxic ROS production [18].

\section{Physiological Roles of Eiger}

Despite its potent ability to activate JNK signaling, the physiological roles of

Eiger have been poorly understood until recently. Recent genetic studies have discovered that Eiger-JNK signaling plays crucial roles in a variety of cellular and tissue 
processes such as tissue growth regulation (including both tumor suppression and tumor progression), host defense, pain sensitization, cell proliferation, and canalization.

\subsection{Tumor-suppressing role of Eiger}

Loss of apico-basal polarity is frequently associated with epithelial tumorigenesis. Indeed, Drosophila imaginal epithelium entirely mutant for evolutionarily conserved apico-basal polarity genes such as scribble (scrib) or discs large (dlg) results in tumorous overgrowth [19], suggesting an active role of polarity disruption in tumor progression. Interestingly, such polarity-deficient oncogenic mutant cells behave in a completely different way when surrounded by wild-type cells.

Clones of cells mutant for scrib or $d l g$ in otherwise wild-type tissue do not overgrow but are eliminated from the tissue by cell death $[20,21]$. This suggests that normal epithelial tissue has an intrinsic tumor suppression mechanism that actively eliminates oncogenic polarity-deficient cells from the tissue. It has been shown that Eiger-JNK signaling plays crucial roles in this intrinsic tumor suppression (Fig. 2A). scrib mutant cells surrounded by wild-type cells elevate endocytosis, which causes translocation of 
Eiger from the plasma membrane to early endosomes. Accumulation of Eiger in endosomes, in turn, triggers apoptotic JNK activation at the endocytic compartment [22] (Fig. 2B), where Eiger might meet adaptor or scaffold proteins that recruit downstream JNK signaling components. Similar endocytic activation of caspase by TNFR signaling has been reported in mammalian systems [23]. Intriguingly, most dying cells were observed at boundaries between scrib mutant clones and wild-type clones, suggesting an apoptotic cell-cell interaction between scrib mutant cells and wild-type cells. Indeed, Eiger-JNK signaling was also found to be specifically activated in wild-type cells nearby polarity-deficient clones. Furthermore, this JNK activation in wild-type cells promotes elimination of their neighboring mutant cells. Mechanistically, JNK activation in wild-type cells surrounding polarity-deficient cells induces expression of PVR, a PDGF/VEGF receptor homolog, which activates the engulfment pathway through actin cytoskeleton regulators ELMO-Mbc (a DOCK180 homolog) [13]. Polarity-deficient cells are thus actively eliminated by engulfment by neighboring wild-type cells (Fig. 2B). These results show that Eiger-JNK signaling in both polarity-deficient cells and surrounding wild-type cells are required for facilitating 
cell elimination, and therefore dying cells were specifically observed at the boundaries between these two populations. It has been shown that this elimination of polarity-deficient clones is the result of 'cell competition' [13, 20, 22, 24], a struggle for existence among cells within the tissue [25]. While Eiger plays a central role in cell competition between scrib mutant cells and wild-type cells, it seems not to be involved in a classical Minute cell competition, a competition between wild-type cells and ribosomal protein mutant cells [13].

Systemic regulation of the elimination of polarity-deficient tumors has also been reported [26]. Eiger expression in imaginal disc tumors triggers activation of Toll signaling in the fat body, which suppresses imaginal tumor growth in a non-tissue autonomous manner. Eiger expression in hemocytes is required for triggering tumor cell death downstream of Toll activation in the fat body.

\subsection{Tumor-promoting role of Eiger}

Contrary to its tumor-suppressor function, Eiger can also function as a tumor-promoter in some contexts. In imaginal discs, clones of cells mutant for 
apico-basal polarity genes such as scrib simultaneously expressing oncogenic $\operatorname{Ras}^{\mathrm{V} 12}$ $\left(\operatorname{Ras}^{\mathrm{V} 12} /\right.$ scrib $^{-/}$clones) result in tumorous overgrowth and metastatic behavior $[20,27]$. JNK signaling activated in $\mathrm{Ras}^{\mathrm{V} 12} / \mathrm{scrib}^{-/-}$clones was shown to play an essential role in tumor growth, as blocking $\mathrm{JNK}$ in $\mathrm{Ras}^{\mathrm{V} 12} / \mathrm{scrib}^{-/-}$clones suppresses tumor growth and clonal activation of Ras and JNK signaling is sufficient to cause tumorous overgrowth [28]. Subsequently, it was shown that Eiger is the upstream trigger for this pro-tumor JNK signaling in $\operatorname{Ras}^{\mathrm{V} 12} /$ scrib $^{-/}$clones [29] (Fig. 3A). Further genetic study has shown that co-activation of Ras and JNK signaling causes tumorous overgrowth through inactivation of the tumor-suppressor Hippo pathway [30]. Thus, Eiger-JNK signaling seems to switch its tumor-suppressor role to a tumor-promoting effect in the presence of oncogenic Ras through regulation of the Hippo pathway (Fig. 3B). Interestingly, it has also been shown that JNK activation in polarity-deficient clones is triggered by Eiger expressed in circulating hemocytes that are recruited to mutant clones [29].

\subsection{Role of Eiger in host defense}

Eiger is also shown to be involved in the process of host defense. It has 
been shown that eiger expression is upregulated after exposure of Drosophila mbn-2

cells to LPS [31]. In addition, Eiger was shown to play a role in regulating the growth

of extracellular pathogens, as eiger mutant flies show decreased phagocytosis that results in exclusion of extracellular pathogens [32]. On the other hand, eiger mutant flies show increased susceptibility to Vibrio choleae infection [33]. eiger mutation does not affect the sensitivity to the intracellular pathogen Salmonella typhimurium but delays host death [34]. In addition, Eiger-JNK signaling was shown to be involved in crystal cell rapture that releases prophenoloxidase [35], a protein required for the process of host defense called melanization.

\subsection{Role of Eiger in pain sensitization}

Nociceptive (pain) sensitivity is crucial for a response to tissue damage to protect against injury. In Drosophila larvae, UV-induced tissue damage develops both thermal hyperalgesia (an exaggerated response to noxious thermal stimuli) and thermal allodynia (a responsiveness to subthreshold thermal stimuli that are not normally noxious). Knockdown of wengen in nociceptive sensory neurons abolished 
development of UV-induced thermal allodynia. In addition, ectopic expression of Eiger in nociceptive sensory neurons was sufficient to induce allodynia even in the absence of UV irradiation. Thus, it was proposed that damaged epidermal cells produce Eiger, which is released from epidermal cells and binds to Wengen on nociceptive sensory neurons underlying the epidermal sheet and lowers the threshold of the behavioral response [36]. Hedgehog signaling was also shown to regulate nociceptive sensitization in the pathway parallel to Eiger/Wengen signaling[37].

\subsection{Role of Eiger in glial proliferation}

During Drosophila development, neuronal cell death occurs in the adult brain shortly after eclosion. Surrounding glial cells respond to this neuronal cell death to undergo cell division, as inhibition of neuronal cell death by the caspase inhibitor p35 suppresses surrounding glial proliferation. This glial cell division was attenuated in eiger mutant flies, whereas cell death soon after eclosion still occurs in mutant flies. Similarly, glial cells undergo cell division in response to brain injury in a manner dependent on Eiger [38]. Although the underlying mechanism is not known, these 
results suggest that Eiger is involved in mitogenic cell-cell communication between apoptotic cells and surrounding viable cells.

\subsection{Role of Eiger in canalization}

The 'constancy of the wild-type' phenotype under varying developmental conditions, termed 'canalization', is achieved by buffering against stochastic fluctuations and environmental perturbations [39]. During dorsal patterning in Drosophila embryo, bone morphogenetic protein (BMP) signaling visualized by pMad staining shows a stable pattern centered on the dorsal midline [40]. Eiger expression is broadly observed in the dorsal region of stage 5 embryos and is restricted to the dorsal most cells at stage $6[2,40,41]$. Eiger expression depends on BMP signaling in the pregastrula embryo. Mean intensity of pMad staining is reduced to half in eiger mutant embryos or in embryos that lack maternal JNK activity. It was shown that Eiger acts in the positive feedback circuit to promote and refine BMP signaling. BMP signaling is antagonized by the BMP binding protein Crossveinless-2 $(\mathrm{Cv}-2)$. zerknullt (zen) homeobox gene, a target of BMP signaling, regulates expression of both eiger and 
$c v-2$. In embryos doubly mutant for eiger and $c v-2$, the variability of BMP signaling became very high, indicating that BMP signaling was 'decanalized'. It has been reported that amnioserosa specification depends on BMP signaling and the variability of amnioserosa cell number is very low in wild-type embryos. This constancy of amnioserosa cell number is also decanalized in eiger, $c v-2$ double mutant embryos. Decanalized status was not affected by higher (29 C) or lower (18 C) temperatures but was affected by maternal heterozygosity for Medea, an obligate BMP transcriptional cofactor. Thus, combined action of eiger and $c v-2$ could canalize BMP signaling against genetic but not environmental fluctuations [40].

\section{Invertebrate and ascidian TNF superfamilies}

TNF ligand and receptor superfamilies have been identified throughout the animal kingdom based on the sequence similarities [42] (Fig. 4). Eiger is the first identified TNF superfamily ligand in invertebrates. Like Eiger in Drosophila, kuruma shrimp (Marsupenaeus japonicus) TNF ligand (MjTNF) is a type II transmembrane protein that shows $30.7 \%$ and $26.7 \%$ identities with Eiger and human EDA, respectively 
[43]. The genome of purple sea urchin, Stronglyocentrotus purpuratus, has four TNF ligands and seven TNFR superfamilies [44]. Ascidian urochordate Ciona intestinalis has two TNF ligands and two TNFRs. Furthermore, sea anemone $N$. vectensis possesses one TNF ligand and two TNFRs [44]. While the genomes of ascidian, sea anemone and sea urchin all contain orthologs of EDA (CiEda in ascidian, NvTNF in sea anemone, Sp-Eda and Sp-Eda2 in sea urchin), they do not have orthologs of Fas, DR4, DR5, or TNFR1, and their ligands FasL, Trail, or TNF- $\alpha$. EDA receptor (EDAR) ortholog was also found in sea urchin. Thus, EDA ligand/receptor members seem to be evolved early during metazoan evolution [44]. Orthologs of $\mathrm{p} 75^{\mathrm{NTR}} / \mathrm{NGFR}$ and its ligand were also found in sea anemone, ascidian and sea urchin [44]. EDAR signaling is important for the development of epithelial appendages, while $\mathrm{p} 75^{\mathrm{NTR}} / \mathrm{NGFR}$ is involved in neuronal survival [45-47]. Throughout the evolution of TNF superfamily members, EDAR and p75 $5^{\mathrm{NTR}} / \mathrm{NGFR}$ subfamilies and their ligands including Eiger might have acquired roles in epithelial and neural development. Although Drosophila genome does not have $\mathrm{p} 75^{\mathrm{NTR}}$ homolog, a recent study on the X-ray structure of dimeric active Spätzle bound to the Toll ectodomain revealed that this ligand/receptor complex 
resembled to $\mathrm{NGF} / \mathrm{p} 75^{\mathrm{NTR}}$ complex rather than microbial ligands bound to Toll like receptor [48, 49]. In mammals, NGF induces oligodendrocyte cell death through $\mathrm{p} 75^{\mathrm{NTR}} / \mathrm{JNK}$ signaling pathway $[50,51]$.

Apart from EDA and $\mathrm{p} 75^{\mathrm{NTR}}$ subfamilies, other TNF superfamilies were cloned from ascidian Ciona savignyi and Ciona intestinalis and named CsTL and CiTNFo, respectively. They show significant homologies with sea urchin $S$. purpuratus Sp-TL1A but not with Drosophila Eiger $[52,53]$. CsTL and CiTNF $\alpha$ are ubiquitously expressed and have been shown to be upregulated in hemocytes after LPS injection, suggesting that these ligands play roles in inflammation and immune response.

A member of the TNF superfamily was also identified in molluscan disk abalone, which was named AbTNF- $\alpha$. AbTNF- $\alpha$ is related to ascidian CsTL and is expressed in immune and non-immune tissues with a highest expression in gill. Another identified TNF superfamily member in molluscan disk abalone is AbFas ligand. AbFas ligand is constitutively expressed in hemocytes, gills, mantle, muscle, digestive tract, and digestive gland. Expressions of AbTNF- $\alpha$ and AbFas ligand are induced in 
gill by pathogenic bacteria, viral haemorrhagic septicaemia virus, or LPS challenge [54,

55]. Expression patterns and the induction by immune stimulation suggest that AbTNF- $\alpha$ and AbFas ligand play roles in the immune system.

TNFR family signaling is mediated by binding of adapter molecules to the cytoplasmic domain of TNFR through its death-domain. Death-domain-containing adaptors such as FADD, TRADD, RAIDD/CRADD, and EDARADD bind homophilically to death-domain of receptors. Drosophila has two death-domain-containing adaptor proteins dFADD and dMyd88. Sea urchin has three death-domain-containing adaptors Sp-FADD, Sp-MyD88, and Sp-RAIDD/Cradd-19. Another adaptor protein family, TRAF family of proteins, binds to death-domain adaptor proteins as well as TNFRs directly [56]. Six TRAF proteins were found in mammals, three were found in Drosophila (dTRAF1, a TRAF4 ortholog; dTRAF3, a precursor for TRAF1, 2, 3 and 5; dTRAF2, a TRAF6 ortholog), and one was found in $C$. elegans $[44,57,58]$. Sea urchin has four TRAFs (Sp-TRAF1/2, Sp-TRAF3/5, Sp-TRAF4 and Sp-TRAF6) [44]. Thus, TRAF4 and TRAF6 orthologs seem to be evolved early during the metazoan evolution. In Drosophila, two TRAFs transduce 
discrete signaling pathways. Overexpression of DTRAF1 causes JNK activation, while overexpression of DTRAF2 leads to activation of Drosophila NF-אB proteins DIF and Relish [59]. Although Wengen can bind to both DTRAF1 and DRTAF2, only JNK signaling, but not the NF-KB pathway, is activated by Eiger. DTRAF1 and DRTAF2 were both reported to be involved in Eiger-induced JNK activation $[5,6]$.

EDA binds to EDAR to form a trimeric ligands/receptor complex, and then the adaptor protein EDARADD is recruited. Subsequently, TAK1 is activated in the TRAF6/TAB2 complex, and TAK1 further activates the IKK complex. This leads to

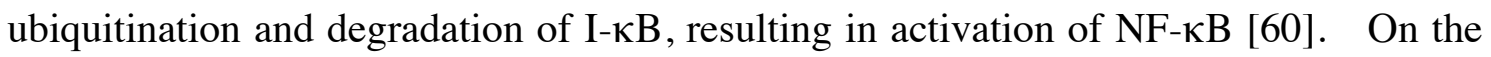
other hand, EDA also activates JNK [61]. Similar receptor complex was found in Eiger/Wengen signaling [5]. dTAK1 was shown to be activated in the complex of dTRAF1/dTAB2/Msn (Fig. 1). However, dTAK1's target is not the IKK complex but Hep, which activates downstream Bsk (JNK).

\section{Evolution of the NF-KB and JNK activation machineries through TNFR}


In mammals, binding of TNF- $\alpha$ to TNFR1 activates membrane-bound signaling complex (complex I), which contains TRADD, TRAF2, cIAP1/2, and RIP1 [62]. Downstream of complex I, IKK complex is activated, which leads to degradation of $\mathrm{I}-\kappa \mathrm{B}$ and subsequent activation of NF- $\mathrm{NB}$. TAK1 is also activated downstream of complex I and triggers p38 and JNK signaling [63]. Complex I endocytosis dissociates TRADD from the complex I, and then FADD is recruited to the complex (this new complex is called complex II). Caspase 8 is recruited to the complex II, thereby initiating the apoptotic pathway.

In Drosophila, binding of Eiger to Wengen activates JNK signaling through

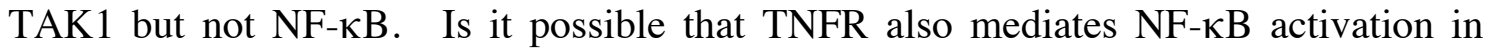
Drosophila? Drosophila cytokine Spätzle/Toll signaling activates NF-кB. Toll is a prototype of Toll-like receptor (TLR) but not a member of the TNFR superfamily. However, the Spätzle/Toll complex resembles to mammalian NGF/p75 ${ }^{\mathrm{NTR}}$ complex [48, 64] and p75 NTR is a member of the TNFR superfamily. Like TNFR1, binding of NGF to $\mathrm{p} 75^{\mathrm{NTR}}$ activates NF-אB [65], JNK [66, 67], and caspase [68]. Thus, two different 


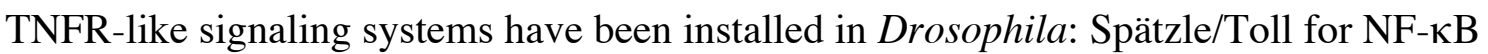
activation and Eiger/Wengen for JNK activation (Fig. 5).

\section{Amplification of inflammatory signaling in invertebrate}

In mammals, upon bacterial infection or exposure of damage-associated molecular patterns (DAMPs), TLR directly senses the bacterial component or DAMPs

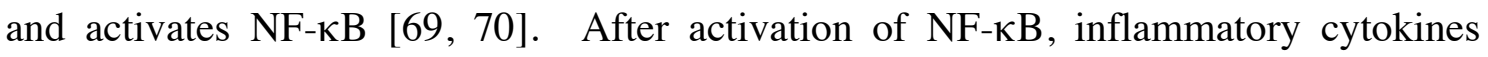
including TNF- $\alpha$ are induced to amplify the inflammatory response. Drosophila has a different strategy to amplify the host inflammatory response against infection or DAMPs exposure through Toll. Proteolytic cascade of CLIP-domain serine protease [71] is utilized to activate proSpätzle after infection [72, 73] or DAMPs stimulation [74]. Spätzle prodomain normally masks the Spätzle's critical region required for binding to Toll, and the processing of the prodomain causes conformational change of Spätzle to be able to bind to Toll [75].

The hemolymph-clotting phenomenon is an efficient defense system found in the horseshoe crab Limulus polyphemus [76]. Studies using other horseshoe crab 
Tachypleus tridentatus have shown that the proteolytic cascade of CLIP-domain serine protease is utilized for hemolymph coagulation upon LPS stimulation [77]. Coagulogen is processed by clotting enzyme and causes its conformational change to form coagulin gel. Structure of coagulogen exhibits a striking topological similarity to NGF and Spätzle [78, 79]. Thus, activation of neurotrophin-fold protein by the conformational changes through proteolytic cascade of CLIP-domain serine protease could be a prototypical signal amplification mechanism for host defense in invertebrate. In Drosophila, Spätzle/Toll, a TNFR-like signaling system, is activated upon necrotic cell death that could be commonly induced by tissue damage or under pathological conditions [80]. Toll activation leads to activation of dFoxO [81], which regulates glycine $\mathrm{N}$-methyltransferase for the maintenance of organismal S-adenosyl-methionine metabolism and energy homeostasis [80]. This invertebrate amplification system for host defense could be widely utilized for sterile inflammation to maintain organismal homeostasis. 


\section{Acknowledgements}

We apologize to colleagues whose work could not be cited owing to space

limitations. We thank S. Iwanaga for stimulative discussion on the evolution of invertebrate immunity. We also thank S. Ohsawa for providing figures. The work in the Igaki and the Miura laboratories were supported in part by KAKENHI from the Japanese Ministry of Education, Science, Sports, Culture, and Technology (MEXT) and by Japan Science and Technology Agency (JST).

\section{References}

[1] Igaki T, Kanda H, Yamamoto-Goto Y, Kanuka H, Kuranaga E, Aigaki T,Miura M. Eiger, a TNF superfamily ligand that triggers the Drosophila JNK pathway. EMBO J 2002;21:3009-18.

[2] Kauppila S, Maaty WS, Chen P, Tomar RS, Eby MT, Chapo J, Chew S, Rathore N, Zachariah S, Sinha SK, Abrams JM, Chaudhary PM. Eiger and its receptor, Wengen, comprise a TNF-like system in Drosophila. Oncogene 2003;22:4860-7.

[3] Moreno E, Yan M,Basler K. Evolution of TNF signaling mechanisms: JNK-dependent apoptosis triggered by Eiger, the Drosophila homolog of the TNF superfamily. Curr Biol 2002;12:1263-8.

[4] Kanda H, Igaki T, Kanuka H, Yagi T,Miura M. Wengen, a member of the Drosophila tumor necrosis factor receptor superfamily, is required for Eiger signaling. J Biol Chem 2002;277:28372-5.

[5] Geuking P, Narasimamurthy R,Basler K. A genetic screen targeting 
the tumor necrosis factor/Eiger signaling pathway: identification of Drosophila TAB2 as a functionally conserved component. Genetics 2005;171:1683-94.

[6] Xue L, Igaki T, Kuranaga E, Kanda H, Miura M,Xu T. Tumor suppressor CYLD regulates JNK-induced cell death in Drosophila. Dev Cell 2007;13:446-54.

[7] Ma X, Huang J, Yang L, Yang Y, Li W,Xue L. NOPO modulates Egr-induced JNK-independent cell death in Drosophila. Cell Res 2012;22:425-31.

[8] Ma X, Li W, Yu H, Yang Y, Li M, Xue L,Xu T. Bendless modulates JNK-mediated cell death and migration in Drosophila. Cell Death Differ 2014;21:407-15.

[9] Ma X, Yang L, Yang Y, Li M, Li W,Xue L. dUev1a modulates TNF-JNK mediated tumor progression and cell death in Drosophila. Dev Biol 2013;380:211-21.

[10] Narasimamurthy R, Geuking P, Ingold K, Willen L, Schneider P,Basler K. Structure-function analysis of Eiger, the Drosophila TNF homolog. Cell Res 2009;19:392-4.

[11] Perez-Garijo A, Fuchs Y,Steller H. Apoptotic cells can induce non-autonomous apoptosis through the TNF pathway. Elife 2013;2:e01004. [12] Wu M, Pastor-Pareja JC,Xu T. Interaction between Ras(V12) and scribbled clones induces tumour growth and invasion. Nature 2010;463:545-8.

[13] Ohsawa S, Sugimura K, Takino K, Xu T, Miyawaki A,Igaki T. Elimination of Oncogenic Neighbors by JNK-Mediated Engulfment in Drosophila. Developmental cell 2011;20:315-28.

[14] Kanda H, Igaki T, Okano H,Miura M. Conserved metabolic energy production pathways govern Eiger/TNF-induced nonapoptotic cell death. Proceedings of the National Academy of Sciences of the United States of America 2011;108:18977-82.

[15] Gottlieb E,Tomlinson IP. Mitochondrial tumour suppressors: a genetic and biochemical update. Nature reviews Cancer 2005;5:857-66. [16] Christofk HR, Vander Heiden MG, Harris MH, Ramanathan A, 
Gerszten RE, Wei R, Fleming MD, Schreiber SL,Cantley LC. The M2 splice isoform of pyruvate kinase is important for cancer metabolism and tumour growth. Nature 2008;452:230-3.

[17] Bonnet S, Archer SL, Allalunis-Turner J, Haromy A, Beaulieu C, Thompson R, Lee CT, Lopaschuk GD, Puttagunta L, Bonnet S, Harry G, Hashimoto K, Porter CJ, Andrade MA, Thebaud B,Michelakis ED. A mitochondria- $\mathrm{K}+$ channel axis is suppressed in cancer and its normalization promotes apoptosis and inhibits cancer growth. Cancer cell 2007;11:37-51.

[18] Kim JW,Dang CV. Cancer's molecular sweet tooth and the Warburg effect. Cancer research 2006;66:8927-30.

[19] Bilder D. Epithelial polarity and proliferation control: links from the Drosophila neoplastic tumor suppressors. Genes Dev 2004;18:1909-25.

[20] Brumby AM,Richardson HE. scribble mutants cooperate with oncogenic Ras or Notch to cause neoplastic overgrowth in Drosophila. EMBO J 2003;22:5769-79.

[21] Woods DF,Bryant PJ. The discs-large tumor suppressor gene of Drosophila encodes a guanylate kinase homolog localized at septate junctions. Cell 1991;66:451-64.

[22] Igaki T, Pastor-Pareja JC, Aonuma H, Miura M,Xu T. Intrinsic tumor suppression and epithelial maintenance by endocytic activation of Eiger/TNF signaling in Drosophila. Dev Cell 2009;16:458-65.

[23] Schneider-Brachert W, Tchikov V, Neumeyer J, Jakob M, Winoto-Morbach S, Held-Feindt J, Heinrich M, Merkel O, Ehrenschwender M, Adam D, Mentlein R, Kabelitz D,Schutze S. Compartmentalization of TNF receptor 1 signaling: internalized TNF receptosomes as death signaling vesicles. Immunity 2004;21:415-28.

[24] Chen CL, Schroeder MC, Kango-Singh M, Tao C,Halder G. Tumor suppression by cell competition through regulation of the Hippo pathway. Proc Natl Acad Sci U S A 2012;109:484-9.

[25] Morata G,Ripoll P. Minutes: mutants of drosophila autonomously affecting cell division rate. Dev Biol 1975;42:211-21.

[26] Parisi F, Stefanatos RK, Strathdee K, Yu Y,Vidal M. Transformed epithelia trigger non-tissue-autonomous tumor suppressor response by 
adipocytes via activation of Toll and Eiger/TNF signaling. Cell Rep 2014;6:855-67.

[27] Pagliarini RA,Xu T. A genetic screen in Drosophila for metastatic behavior. Science 2003;302:1227-31.

[28] Igaki T, Pagliarini RA,Xu T. Loss of cell polarity drives tumor growth and invasion through JNK activation in Drosophila. Curr Biol 2006;16:1139-46.

[29] Cordero JB, Macagno JP, Stefanatos RK, Strathdee KE, Cagan RL,Vidal M. Oncogenic Ras diverts a host TNF tumor suppressor activity into tumor promoter. Developmental cell 2010;18:999-1011.

[30] Ohsawa S, Sato Y, Enomoto M, Nakamura M, Betsumiya A,Igaki T. Mitochondrial defect drives non-autonomous tumour progression through Hippo signalling in Drosophila. Nature 2012;490:547-51.

[31] Johansson KC, Metzendorf C,Soderhall K. Microarray analysis of immune challenged Drosophila hemocytes. Exp Cell Res 2005;305:145-55. [32] Schneider DS, Ayres JS, Brandt SM, Costa A, Dionne MS, Gordon MD, Mabery EM, Moule MG, Pham LN,Shirasu-Hiza MM. Drosophila eiger mutants are sensitive to extracellular pathogens. PLoS Pathog 2007;3:e41. [33] Berkey CD, Blow N,Watnick PI. Genetic analysis of Drosophila melanogaster susceptibility to intestinal Vibrio cholerae infection. Cell Microbiol 2009;11:461-74.

[34] Brandt SM, Dionne MS, Khush RS, Pham LN, Vigdal TJ,Schneider DS. Secreted Bacterial Effectors and Host-Produced Eiger/TNF Drive Death in aSalmonella-Infected Fruit Fly. PLoS Biol 2004;2:e418.

[35] Bidla G, Dushay MS,Theopold U. Crystal cell rupture after injury in Drosophila requires the JNK pathway, small GTPases and the TNF homolog Eiger. J Cell Sci 2007;120:1209-15.

[36] Babcock DT, Landry C,Galko MJ. Cytokine signaling mediates UV-induced nociceptive sensitization in Drosophila larvae. Curr Biol 2009;19:799-806.

[37] Babcock DT, Shi S, Jo J, Shaw M, Gutstein HB,Galko MJ. Hedgehog signaling regulates nociceptive sensitization. Curr Biol 2011;21:1525-33. 
[38] Kato K, Awasaki T,Ito K. Neuronal programmed cell death induces glial cell division in the adult Drosophila brain. Development 2009;136:51-9. [39] Waddington CH. CANALIZATION OF DEVELOPMENT AND THE INHERITANCE OF ACQUIRED CHARACTERS. Nature 1942;150:563-65. [40] Gavin-Smyth J, Wang YC, Butler I,Ferguson EL. A genetic network conferring canalization to a bistable patterning system in Drosophila. Curr Biol 2013;23:2296-302.

[41] Stathopoulos A, Van Drenth M, Erives A, Markstein M,Levine M. Whole-genome analysis of dorsal-ventral patterning in the Drosophila embryo. Cell 2002;111:687-701.

[42] Wiens GD,Glenney GW. Origin and evolution of TNF and TNF receptor superfamilies. Dev Comp Immunol 2011;35:1324-35.

[43] Mekata T, Sudhakaran R, Okugawa S, Inada M, Kono T, Sakai M,Itami T. A novel gene of tumor necrosis factor ligand superfamily from kuruma shrimp, Marsupenaeus japonicus. Fish Shellfish Immunol 2010;28:571-8.

[44] Robertson AJ, Croce J, Carbonneau S, Voronina E, Miranda E, McClay DR,Coffman JA. The genomic underpinnings of apoptosis in Strongylocentrotus purpuratus. Developmental biology 2006;300:321-34. [45] Colosimo PF, Hosemann KE, Balabhadra S, Villarreal G, Jr., Dickson M, Grimwood J, Schmutz J, Myers RM, Schluter D,Kingsley DM. Widespread parallel evolution in sticklebacks by repeated fixation of Ectodysplasin alleles. Science 2005;307:1928-33.

[46] Bridgham JT, Wilder JA, Hollocher H,Johnson AL. All in the family: evolutionary and functional relationships among death receptors. Cell death and differentiation 2003;10:19-25.

[47] Lobito AA, Gabriel TL, Medema JP,Kimberley FC. Disease causing mutations in the TNF and TNFR superfamilies: Focus on molecular mechanisms driving disease. Trends in molecular medicine 2011;17:494-505. [48] Lewis M, Arnot CJ, Beeston H, McCoy A, Ashcroft AE, Gay NJ,Gangloff M. Cytokine Spatzle binds to the Drosophila immunoreceptor Toll with a neurotrophin-like specificity and couples receptor activation. Proc Natl Acad Sci U S A 2013;110:20461-6. 
[49] Parthier C, Stelter M, Ursel C, Fandrich U, Lilie H, Breithaupt C,Stubbs MT. Structure of the Toll-Spatzle complex, a molecular hub in Drosophila development and innate immunity. Proc Natl Acad Sci U S A 2014;111:6281-6.

[50] Harrington AW, Kim JY,Yoon SO. Activation of Rac GTPase by p75 is necessary for c-jun N-terminal kinase-mediated apoptosis. The Journal of neuroscience : the official journal of the Society for Neuroscience 2002;22:156-66.

[51] Casaccia-Bonnefil P, Carter BD, Dobrowsky RT, Chao MV. Death of oligodendrocytes mediated by the interaction of nerve growth factor with its receptor p75. Nature 1996;383:716-9.

[52] Zhang X, Luan W, Jin S,Xiang J. A novel tumor necrosis factor ligand superfamily member (CsTL) from Ciona savignyi: molecular identification and expression analysis. Dev Comp Immunol 2008;32:1362-73. [53] Parrinello N, Vizzini A, Arizza V, Salerno G, Parrinello D, Cammarata M, Giaramita FT,Vazzana M. Enhanced expression of a cloned and sequenced Ciona intestinalis TNFalpha-like (CiTNF alpha) gene during the LPS-induced inflammatory response. Cell and tissue research 2008;334:305-17.

[54] De Zoysa M, Jung S,Lee J. First molluscan TNF-alpha homologue of the TNF superfamily in disk abalone: molecular characterization and expression analysis. Fish Shellfish Immunol 2009;26:625-31.

[55] De Zoysa M, Nikapitiya C, Moon DO, Whang I, Kim GY,Lee J. A novel Fas ligand in mollusk abalone: molecular characterization, immune responses and biological activity of the recombinant protein. Fish Shellfish Immunol 2009;27:423-32.

[56] Inoue J, Ishida T, Tsukamoto N, Kobayashi N, Naito A, Azuma $\mathrm{S}$,Yamamoto T. Tumor necrosis factor receptor-associated factor (TRAF) family: adapter proteins that mediate cytokine signaling. Experimental cell research 2000;254:14-24.

[57] Wajant H, Grell M,Scheurich P. TNF receptor associated factors in cytokine signaling. Cytokine \& growth factor reviews 1999;10:15-26.

[58] Grech A, Quinn R, Srinivasan D, Badoux X,Brink R. Complete 
structural characterisation of the mammalian and Drosophila TRAF genes: implications for TRAF evolution and the role of RING finger splice variants. Molecular immunology 2000;37:721-34.

[59] Cha GH, Cho KS, Lee JH, Kim M, Kim E, Park J, Lee SB,Chung J. Discrete functions of TRAF1 and TRAF2 in Drosophila melanogaster mediated by c-Jun N-terminal kinase and NF-kappaB-dependent signaling pathways. Molecular and cellular biology 2003;23:7982-91.

[60] Sadier A, Viriot L, Pantalacci S,Laudet V. The ectodysplasin pathway: from diseases to adaptations. Trends in genetics : TIG 2014;30:24-31.

[61] Kumar A, Eby MT, Sinha S, Jasmin A,Chaudhary PM. The ectodermal dysplasia receptor activates the nuclear factor-kappaB, JNK, and cell death pathways and binds to ectodysplasin A. J Biol Chem 2001;276:2668-77.

[62] Puimege L, Libert C,Van Hauwermeiren F. Regulation and dysregulation of tumor necrosis factor receptor-1. Cytokine \& growth factor reviews 2014;

[63] Sabio G,Davis RJ. TNF and MAP kinase signalling pathways. Seminars in immunology 2014;

[64] Parthier C, Stelter M, Ursel C, Fandrich U, Lilie H, Breithaupt C,Stubbs MT. Structure of the Toll-Spatzle complex, a molecular hub in Drosophila development and innate immunity. Proceedings of the National Academy of Sciences of the United States of America 2014;

[65] Carter BD, Kaltschmidt C, Kaltschmidt B, Offenhauser N, Bohm-Matthaei R, Baeuerle PA,Barde YA. Selective activation of NF-kappa $B$ by nerve growth factor through the neurotrophin receptor $\mathrm{p} 75$. Science 1996;272:542-5.

[66] Friedman WJ. Neurotrophins induce death of hippocampal neurons via the p75 receptor. The Journal of neuroscience : the official journal of the Society for Neuroscience 2000;20:6340-6.

[67] Yoon SO, Casaccia-Bonnefil P, Carter B,Chao MV. Competitive signaling between TrkA and p75 nerve growth factor receptors determines cell survival. The Journal of neuroscience : the official journal of the Society 
for Neuroscience 1998;18:3273-81.

[68] Troy CM, Friedman JE,Friedman WJ. Mechanisms of p75-mediated death of hippocampal neurons. Role of caspases. The Journal of biological chemistry 2002;277:34295-302.

[69] Kang JY,Lee JO. Structural biology of the Toll-like receptor family. Annual review of biochemistry 2011;80:917-41.

[70] Kawai T,Akira S. Toll-like receptors and their crosstalk with other innate receptors in infection and immunity. Immunity 2011;34:637-50.

[71] Muta T, Hashimoto R, Miyata T, Nishimura H, Toh Y,Iwanaga S. Proclotting enzyme from horseshoe crab hemocytes. cDNA cloning, disulfide locations, and subcellular localization. The Journal of biological chemistry 1990;265:22426-33.

[72] Valanne S, Wang JH,Ramet M. The Drosophila Toll signaling pathway. Journal of immunology 2011;186:649-56.

[73] Jang IH, Nam HJ,Lee WJ. CLIP-domain serine proteases in Drosophila innate immunity. BMB reports 2008;41:102-7.

[74] Ming M, Obata F, Kuranaga E,Miura M. Persephone/Spatzle pathogen sensors mediate the activation of Toll receptor signaling in response to endogenous danger signals in apoptosis-deficient Drosophila. The Journal of biological chemistry 2014;289:7558-68.

[75] Arnot CJ, Gay NJ,Gangloff M. Molecular mechanism that induces activation of Spatzle, the ligand for the Drosophila Toll receptor. The Journal of biological chemistry 2010;285:19502-9.

[76] Bang FB. A bacterial disease of Limulus polyphemus. Bulletin of the Johns Hopkins Hospital 1956;98:325-51.

[77] Muta T,Iwanaga S. The role of hemolymph coagulation in innate immunity. Current opinion in immunology 1996;8:41-7.

[78] Bergner A, Oganessyan V, Muta T, Iwanaga S, Typke D, Huber R,Bode W. Crystal structure of a coagulogen, the clotting protein from horseshoe crab: a structural homologue of nerve growth factor. The EMBO journal 1996;15:6789-97.

[79] Bergner A, Muta T, Iwanaga S, Beisel HG, Delotto R,Bode W. Horseshoe crab coagulogen is an invertebrate protein with a nerve growth 
factor-like domain. Biological chemistry 1997;378:283-7.

[80] Obata F, Kuranaga E, Tomioka K, Ming M, Takeishi A, Chen CH, Soga T,Miura M. Necrosis-Driven Systemic Immune Response Alters SAM Metabolism through the FOXO-GNMT Axis. Cell Rep 2014;

[81] DiAngelo JR, Bland ML, Bambina S, Cherry S,Birnbaum MJ. The immune response attenuates growth and nutrient storage in Drosophila by reducing insulin signaling. Proc Natl Acad Sci U S A 2009;106:20853-8. [82] Miller DJ, Hemmrich G, Ball EE, Hayward DC, Khalturin K, Funayama N, Agata K,Bosch TC. The innate immune repertoire in cnidaria--ancestral complexity and stochastic gene loss. Genome Biol 2007;8:R59.

[83] Mukherjee K, Korithoski B,Kolaczkowski B. Ancient origins of vertebrate-specific innate antiviral immunity. Mol Biol Evol 2013;31:140-53. 


\section{Figure legends}

Figure 1. Eiger-induced signaling that regulates a variety of cellular and tissue processes. See text for details.

Figure 2. Intrinsic tumor suppression by Eiger-JNK signaling. (A) Clones of cells mutant for scrib are eliminated from the imaginal epithelium in a manner dependent on Eiger-JNK signaling. (B) Polarity-deficient cells surrounded by wild-type cells cause endocytic activation of apoptotic Eiger-JNK signaling, while surrounding wild-type cells activate the engulfment pathway through Eiger-JNK signaling,

Figure 3. Eiger-JNK signaling switches its tumor-suppressor role to a tumor-promoting effect in the presence of oncogenic Ras. (A) Clones of cells mutant for scrib simultaneously expressing oncogenic Ras ${ }^{\mathrm{V} 12}$ result in tumor growth and invasion. (B) Ras signaling switches Eiger-JNK signaling from tumor-suppressor to tumor-promoting effect through regulation of the Hippo pathway. 
Figure 4. Evolution of the TNF superfamily. The numbers of TNF superfamily ligands, receptors, and adaptor molecules associated with receptors are shown in each phylogenetic tree. The number in parenthesis indicate the number of receptors or adaptors with death-domains. The figure is adapted from $[1,3,82,83]$.

Figure 5. The Human and Drosophila TNF systems. In humans, EDAR and $\mathrm{p} 75^{\mathrm{NTR}}$ activate both NF-кB and JNK signaling upon ligand binding. In Drsophlia, EDA/EDAR ortholog Eiger/Wengen activates only JNK signaling. Spätzle/Toll, a structural homolog of NGF/p $75^{\mathrm{NTR}}$, activates only NF-אB signaling. 
Figure 1

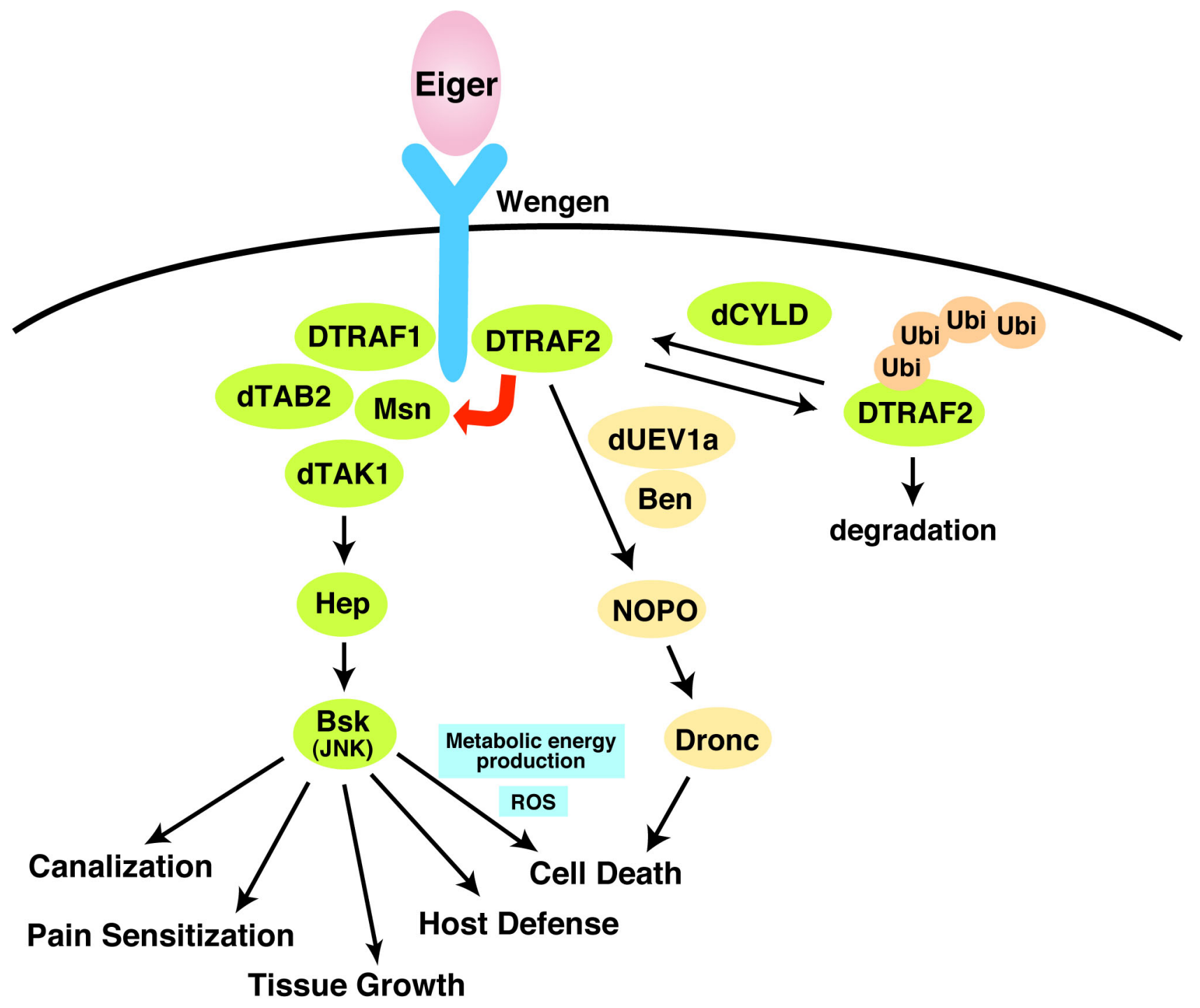


Figure 2

A

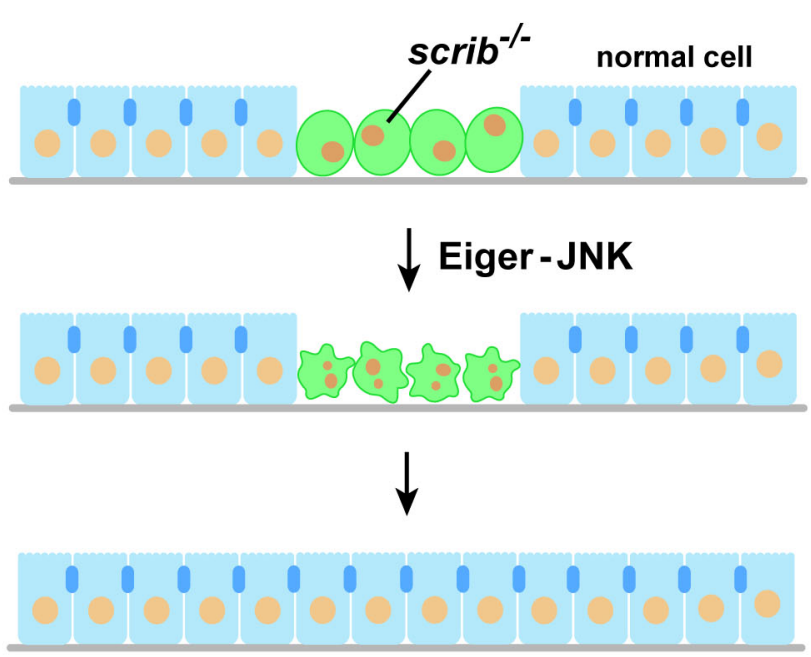

B

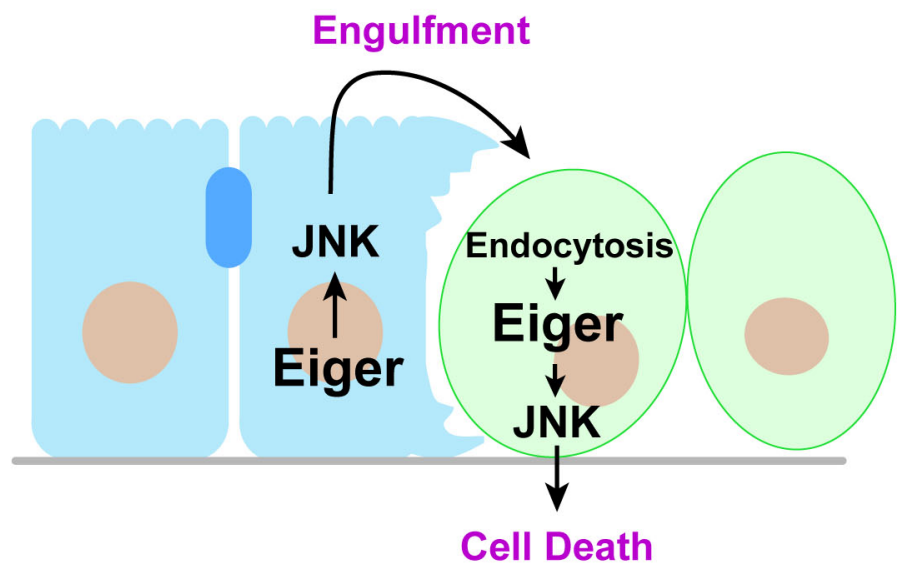


Figure 3

A
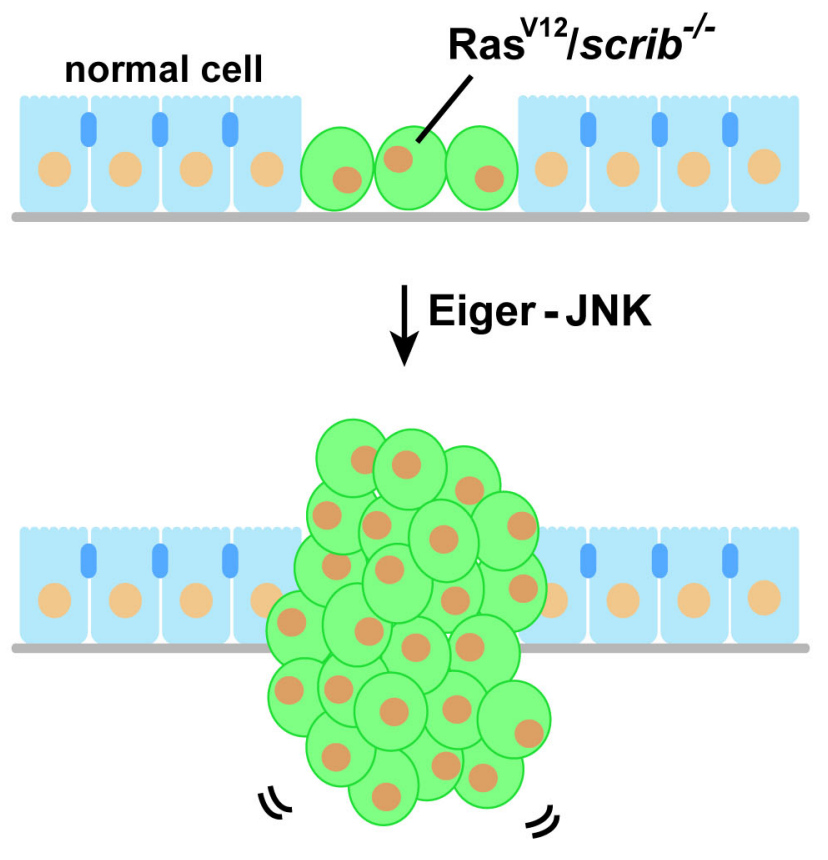

Tumor Growth \& Invasion
B

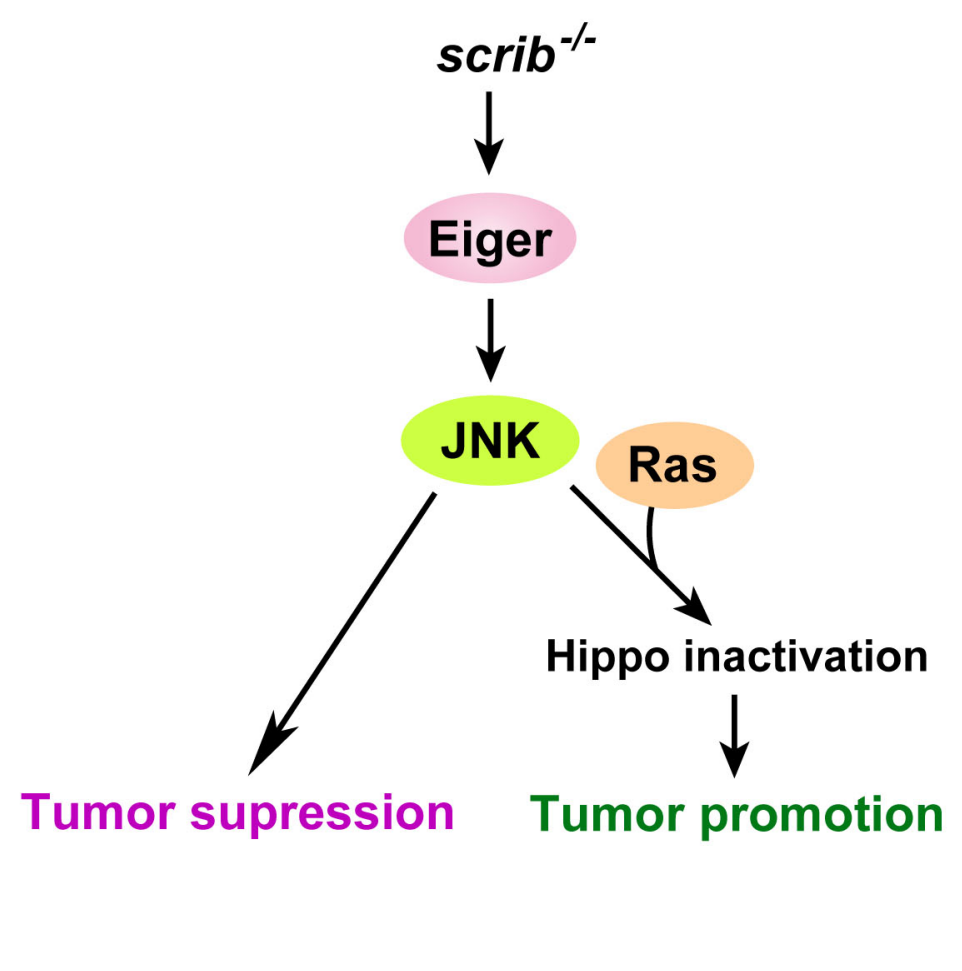


Figure 4

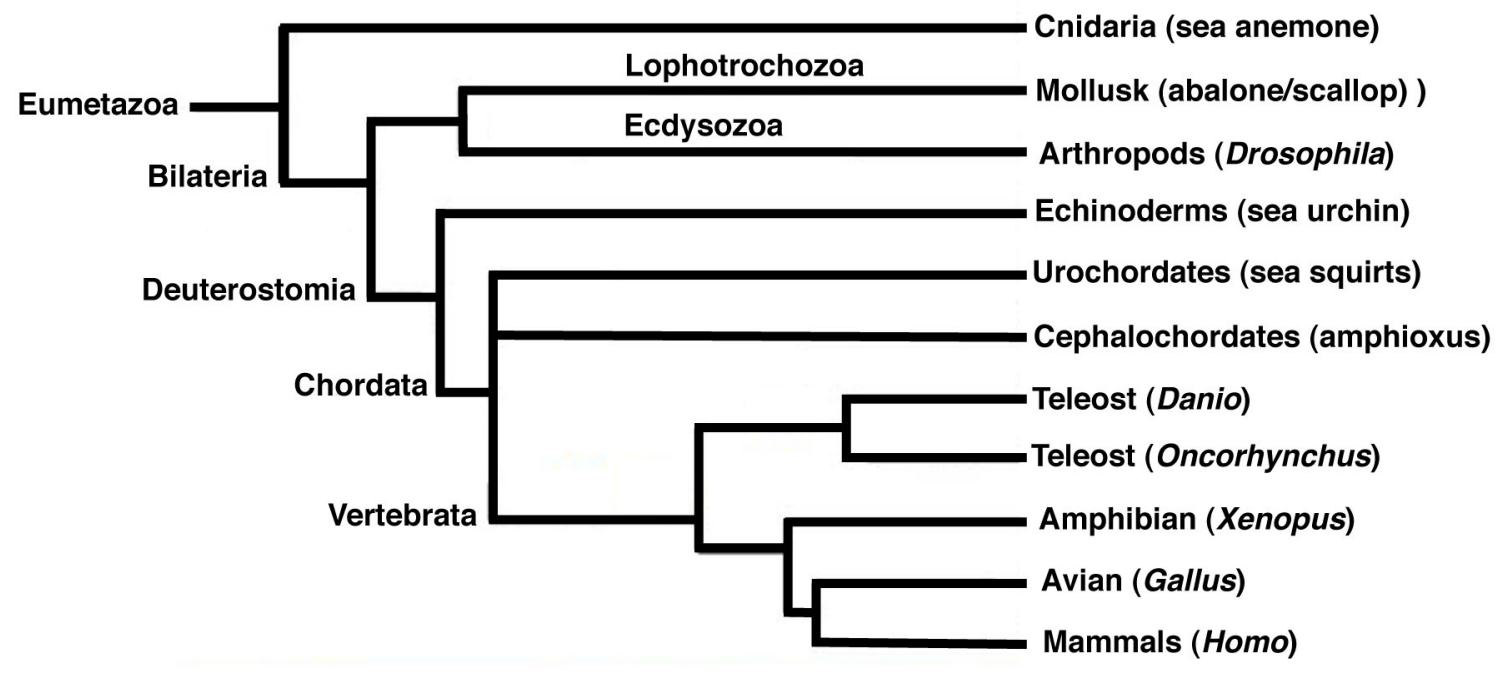

TNF TNF Adaptors ligands receptors

$\begin{array}{rcc}>1 & 1(1) & 6(2) \\ 2 & 1(1) & \\ 1 & 1(0) & 5(2) \\ 4 & 7(3) & 7(3) \\ 2 & 2(1) & \\ ? & >2(?) & \\ 17 & \sim 33(8) & \\ >11 & ? \\ 13 & >3(3) & \\ 10 & 11(4) & \\ 18 & 29(8) & 11(5)\end{array}$


Figure 5
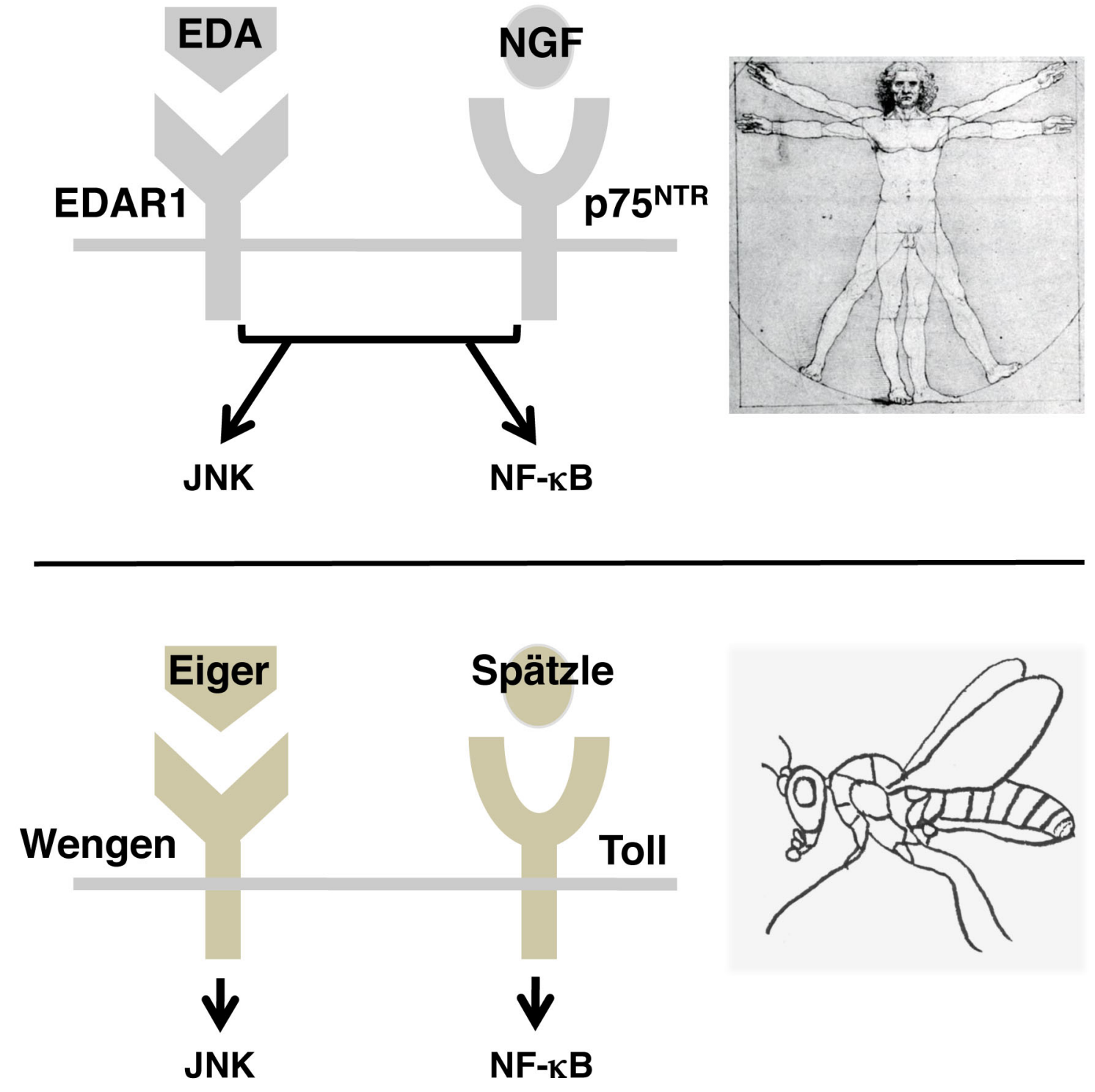